\title{
Effects of Repeated Oral Administration of Esaxerenone on the Pharmacokinetics of Midazolam in Healthy Japanese Males
}

\author{
Kaoru Toyama ${ }^{1} \cdot$ Hidetoshi Furuie $^{2} \cdot$ Kana Kuroda $^{1} \cdot$ Tomoko Ishizuka $^{1} \cdot$ Yasuyuki Okuda ${ }^{1} \cdot$ Takako Shimizu $^{1}$. \\ Manabu Kato ${ }^{1} \cdot$ Yoshiyuki Igawa $^{1} \cdot$ Yasuhiro Nishikawa ${ }^{1} \cdot$ Hitoshi Ishizuka $^{1}$
}

Accepted: 24 June 2021 / Published online: 12 August 2021

(C) The Author(s) 2021

\begin{abstract}
Background and Objective Esaxerenone showed the potential to inhibit and induce activity against cytochrome P450 (CYP) $3 \mathrm{~A}$ in in vitro studies. We investigated whether repeated administration of $5 \mathrm{mg} / \mathrm{day}$ esaxerenone for 14 days influences the pharmacokinetics of midazolam, a sensitive CYP3A substrate, in healthy Japanese males.

Methods This single-centre, open-label, single-sequence study had two administration periods: period 1: single oral dose of $2 \mathrm{mg}$ midazolam (day 0); period 2: repeated oral doses of $5 \mathrm{mg}$ /day esaxerenone for 14 days, with a single oral dose of $2 \mathrm{mg}$ midazolam on day 14. Full pharmacokinetic profiles of midazolam and 1-hydroxymidazolam on days 0 and 14 and safety data were obtained. Primary pharmacokinetic endpoints for midazolam were area under the plasma concentrationtime curve (AUC) from zero to time of the last measurable concentration $\left(\mathrm{AUC}_{\text {last }}\right), \mathrm{AUC}$ from zero to infinity $\left(\mathrm{AUC}_{\text {inf }}\right)$, and peak plasma concentration $\left(C_{\max }\right)$.

Results The study included 28 male subjects. One subject was withdrawn because of a mild adverse event (increased hepatic enzyme levels) that resolved without intervention. Repeated administration of esaxerenone increased midazolam $\mathrm{AUC}_{\text {last }}$, $\mathrm{AUC}_{\mathrm{inf}}$, and $C_{\max }$ by about 1.2-fold (1.201,1.201, and 1.224, respectively) compared with administration of midazolam alone. However, repeated administration of esaxerenone did not affect the elimination half-life of midazolam (2.86 versus $2.63 \mathrm{~h}$ with and without esaxerenone). There were no safety concerns associated with concomitant administration of esaxerenone and midazolam.

Conclusions Esaxerenone $5 \mathrm{mg} /$ day had no clinically significant effect on midazolam pharmacokinetics and was not associated with any safety issues. Esaxerenone can be concomitantly administered with drugs of CYP3A substrates without dose adjustments.
\end{abstract}

Clinical trial registration JapiCTI-152832.

\section{Introduction}

Uncontrolled hypertension is associated with vasculopathy, heart disease, cerebrovascular disease, and nephropathy, all of which typify hypertensive end-organ damage [1]. Antihypertensive therapy has been proven to reduce cardiovascular morbidity and mortality [2]. However, patients with hypertension whose blood pressure (BP) cannot be adequately

Kaoru Toyama

toyama.kaoru.fb@daiichisankyo.jp

Daiichi Sankyo Co., Ltd., Tokyo, Japan

2 Osaka Pharmacology Clinical Research Hospital, Osaka, Japan controlled with a single agent usually receive more than one antihypertensive [3-5].

In Japan, most patients with hypertension are treated with more than one antihypertensive agent [6]. $\mathrm{Ca}^{2+}$-channel blockers (e.g. amlodipine) are particularly widely used, and Japanese Guidelines for the Management of Hypertension (JSH2019) recommend the addition of an aldosterone blocker, or other drugs, to the antihypertensive schedule in patients with uncontrolled or refractory hypertension [3]. Several studies have demonstrated benefit for steroidal mineralocorticoid receptor (MR) blockers, such as spironolactone and eplerenone, in the treatment of refractory hypertension [7-9]. However, there are some limitations with the use of spironolactone and eplerenone; spironolactone causes side effects related to sex hormones, and eplerenone 


\section{Key Points}

The drug-drug interaction of esaxerenone with midazolam in relation to cytochrome P450 (CYP) 3A was evaluated in a clinical study after in vitro studies revealed that esaxerenone has the potential to inhibit and induce activity against CYP3A

Administration of $5 \mathrm{mg} /$ day esaxerenone for 14 days produced an approximately 1.2-fold increase in area under the plasma concentration-time curve (AUC) of midazolam. The elimination half-life of midazolam was unaffected by esaxerenone

Esaxerenone did not meet the threshold criterion for weak inhibition of CYP3A (1.25-2.0-fold increase in midazolam AUC). Esaxerenone at $5 \mathrm{mg} /$ day is unlikely to be associated with clinically significant drug-drug interactions related to CYP3A

is contraindicated in diabetic patients with albuminuria and moderate renal impairment [10].

Esaxerenone (CS-3150) is a novel, oral, non-steroidal, selective MR blocker approved for the treatment of hypertension and is currently in development for the treatment of diabetic nephropathy in Japan [11]. The starting dosage is usually $2.5-5.0 \mathrm{mg} / \mathrm{day}$, but in some patients who require careful consideration, such as patients with moderate renal impairment (estimated glomerular filtration rate: 30 to $<60 \mathrm{ml} / \mathrm{min} / 1.73 \mathrm{~m}^{2}$ ) and diabetes patients with albuminuria or proteinuria, the starting dosage is $1.25 \mathrm{mg} /$ day. Preclinical studies in rats showed that esaxerenone inhibited BP increases because of deoxycorticosterone acetate or saltloading with cardiorenal organ protection [12-15]. Safety and tolerability of esaxerenone were confirmed in phase 1 studies, in which a total of 87 healthy Japanese adults received single (5-200 $\mathrm{mg}$ ) or multiple (10-100 $\mathrm{mg}$ /day for 10 days) esaxerenone doses, which have shown no significant safety- or tolerability-related problems [16]. Esaxerenone ( 2.5 and $5 \mathrm{mg} /$ day) was also shown to be as effective as eplerenone $(50 \mathrm{mg} /$ day) in a phase 3 study in Japanese patients with essential hypertension: esaxerenone $2.5 \mathrm{mg} /$ day was non-inferior to eplerenone in reducing sitting and 24-h BP, and the proportions of patients achieving target sitting $\mathrm{BP}(<140 / 90 \mathrm{mmHg}$ ) were $31.5 \%$ (esaxerenone $2.5 \mathrm{mg} /$ day), $41.2 \%$ (esaxerenone $5 \mathrm{mg} /$ day), and $27.5 \%$ (eplerenone $50 \mathrm{mg} /$ day) [17].

Polypharmacy is common in patients receiving antihypertensive drugs; thus, potential drug interactions with esaxerenone should be considered in the management of these patients. Many antihypertensive agents such as $\mathrm{Ca}^{2+}$-channel blockers are metabolised by cytochrome P450 (CYP) isozyme 3A [18, 19], which creates the potential for drugdrug interactions (DDIs) [20, 21]. Many antihypertensive agents other than $\mathrm{Ca}^{2+}$-channel blockers (e.g. bisoprolol, carvedilol, eplerenone, or indapamide) are also metabolised by CYP3A, again introducing potential for DDIs [22]. In an in vitro study using fresh human hepatocytes, esaxerenone demonstrated CYP3A-inducing activity, by increasing mRNA levels and enzymatic activity of CYP3A4, although these effects were weaker than those of the rifampicin control, and in human liver microsomes, esaxerenone showed weak, time-dependent inhibitory activity against CYP3A [23]. These findings suggest that coadministration of esaxerenone with CYP3A substrates may lead to altered plasma CYP3A-substrate concentrations. Thus, it is clinically important to evaluate the potential for CYP3A-mediated DDIs associated with esaxerenone administration in humans. Midazolam, a benzodiazepine sedative-hypnotic agent, is an index substrate for CYP3A. It undergoes metabolism by CYP3A and is associated with numerous DDIs. Midazolam is recommended as an index substrate for DDI testing and has been widely used as an indicator of CYP3A activity in humans [24-28].

The purpose of the current study was to investigate whether repeated oral administration of $5 \mathrm{mg}$ /day esaxerenone influences the pharmacokinetics of a single oral dose of midazolam, a sensitive CYP3A substrate, in healthy Japanese male subjects.

\section{Methods}

\subsection{Study Design}

This was a single-centre, open-label, single-sequence study with a target sample size of 28 subjects. The study had two administration periods: period $1-$ a single dose of midazolam (day 0); and period 2-repeated doses of $5 \mathrm{mg}$ /day esaxerenone for 14 days, with administration of a single dose of midazolam on day 14 (Fig. 1). Single $2 \mathrm{mg}$ oral doses of midazolam syrup $(1 \mathrm{ml})$ were administered on days 0 and 14 after subjects had fasted for $\geq 10 \mathrm{~h}$. All subjects received $5 \mathrm{mg} /$ day esaxerenone $(2 \times 2.5 \mathrm{mg}$ tablets once daily) from day 1 to day 14. Esaxerenone was administered under fasting conditions on day 1 and after breakfast on days 2-13 and was co-administered with $2 \mathrm{mg}$ midazolam syrup (1 ml) under fasting conditions on day 14. Breakfast on days 2-13 consisted of an ordinary diet (total 500-600 kcal, with fats representing $20-30 \%$ of total calories). Breakfast was completed within $20 \mathrm{~min}$, and esaxerenone was administered with $200 \mathrm{ml}$ water at $30 \mathrm{~min}$ after breakfast completion. From $1 \mathrm{~h}$ before to $2 \mathrm{~h}$ after study drug administration, ingestion of water was prohibited, except for $200 \mathrm{ml}$ 
at the time of administration on days 0,1 , and 14 . Subjects were sitting at rest at the time of study drug administration until $4 \mathrm{~h}$ after administration on days 0,1 , and 14. All subjects were followed up for 6-8 days after the last study drug administration.

The esaxerenone dose was set as $5 \mathrm{mg}$, as this was the maximum dose evaluated in a completed phase 3 study and is now the maximum approved dose for the treatment of hypertension $[11,17]$. The safety and tolerability of $100 \mathrm{mg}$ esaxerenone administered over 10 days in repeated doses were confirmed in an earlier phase 1 study in healthy Japanese adult males [16]. Administration during period 2 in the current study was based on the time to plateauing of plasma esaxerenone concentrations in the earlier phase 1 study [16].

The study was conducted in accordance with Good Clinical Practice and the ethical principles of the Declaration of Helsinki and the Pharmaceuticals, Medical Devices, and Other Therapeutic Products Act. The study was conducted after review and approval by the institutional review board of Osaka Pharmacology Clinical Research Hospital, Osaka, Japan (control number of the study site 930PC). All subjects provided written informed consent to be included in the study (JapicCTI No. 152832).

\subsection{Study Subjects}

All subjects were Japanese males aged 20-45 years, who had a body mass index (BMI) of $18.5-24.9 \mathrm{~kg} / \mathrm{m}^{2}$, sitting $\mathrm{BP}$ of $<140 / 90 \mathrm{mmHg}$, and a pulse rate of $\leq 99$ beats $/ \mathrm{min}$.

The key exclusion criteria were as follows: any previous, serious disease considered by an investigator to affect the study or a disease for which midazolam is contraindicated (e.g. acute narrow-angle glaucoma); drug hypersensitivity or idiosyncratic reactions; drug or alcohol dependence; positive infection test result; previous participation in a clinical study of esaxerenone; required concomitant therapy that induces CYP3A4 or taken within 30 days before the study; having any finding considered clinically significant at screening; and being judged ineligible for participation in the study.

\subsection{Pharmacokinetic Assessments and Endpoints}

Blood samples were collected for analysis of plasma concentrations of midazolam and its metabolite (1-hydroxymidazolam) before and $0.25,0.5,1,1.5,2,3,4,6,8,10,12$, 16, and $24 \mathrm{~h}$ after administration on days 0 and 14 (Fig. 1). Blood samples were collected for analysis of plasma esaxerenone concentrations before and 1, 2, 3, 4, 5, 6, 8, 10, 12, 16, and $24 \mathrm{~h}$ after administration on day 1 ; the same schedule was followed on day 14, except for additional collections 36 and $48 \mathrm{~h}$ after esaxerenone administration.

For measurement of plasma esaxerenone concentration, $3 \mathrm{ml}$ of venous blood from a cephalic vein in each subject's forearm was collected in K2-EDTA tubes. For measurement of plasma midazolam concentration, $7 \mathrm{ml}$ of venous blood from a cephalic vein in each subject's forearm was collected in a sodium heparinized blood collection tube. After blood collection, tubes were mixed by inversion immediately and then cooled with ice. Plasma obtained by centrifugation $\left(4{ }^{\circ} \mathrm{C}, 1700 \mathrm{~g}, 10 \mathrm{~min}\right)$ was fractionated into two storage tubes $(0.5 \mathrm{ml}$ for the esaxerenone measurement in one tube, with the remaining amount in the other tube) and was frozen until measurement. For measurement of plasma midazolam concentration, about $1.5 \mathrm{ml}$ (and the remaining plasma) was fractionated into two storage containers and frozen.

The details of bioanalytical methods have been described previously [16]. Midazolam, 1-hydroxymidazolam, and their internal standards were extracted from plasma samples $(200 \mu \mathrm{l})$ through liquid-liquid extraction. Chromatographic separation was performed using a ZORBAX Eclipse PLUS

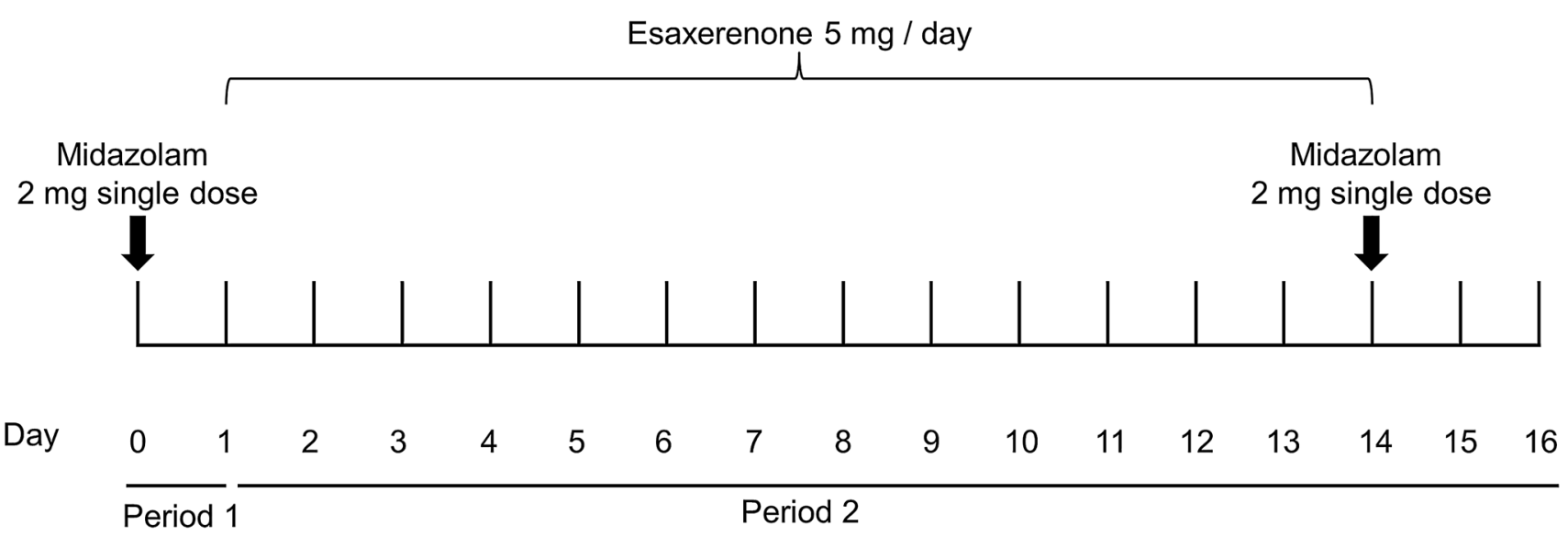

Fig. 1 Study design 
C18 Rapid Resolution HT (2.1 mm $\times 50$ mm, $1.8 \mu \mathrm{m}$, Agilent, CA, USA). Detection was performed by an AB SCIEX 4000 (AB SCIEX, Framingham, MA, USA) tandem mass spectrometer with TurboIonSpray source by electrospray ionization in the positive ion mode and multiple-reaction monitoring of midazolam $(\mathrm{m} / \mathrm{z}$ 326.1-291.1), 1-hydroxymidazolam $(\mathrm{m} / \mathrm{z} 342.1-324.1)$, and their internal standards (midazolam-d4 [m/z 330.1-295.1] and 1-hydroxymidazolam-d4 [m/z 346.1-328.1]). The assay accuracy was in the range of $2.68-9.36 \%$ for midazolam and $3.44-5.11 \%$ for 1-hydroxymidazolam, and the lower limit of quantification was $0.1 \mathrm{ng} / \mathrm{ml}$ for both analytes. Esaxerenone and its internal standard (d7-form) were extracted from plasma samples $(50 \mu \mathrm{l})$ and chromatographic separation was performed using a CAPCELL PAK C18 MGIII (Shiseido Co., Ltd., Tokyo, Japan) column $(2.0 \times 150 \mathrm{~mm}, 5 \mu \mathrm{m})$. Detection was performed using a Sciex API 4000 (AB SCIEX) tandem mass spectrometer with multiple-reaction monitoring of esaxerenone $(\mathrm{m} / \mathrm{z} 465-365)$ and its internal standard $(\mathrm{m} / \mathrm{z}$ $472-370)$. The assay accuracy was in the range of -1.0 to $0.3 \%$, and the lower limit of quantification was $0.1 \mathrm{ng} / \mathrm{ml}$.

Drug concentration measurements that were missing because samples could not be collected or because measurements could not be obtained because of sample processing problems were not imputed. The following pharmacokinetic parameters were calculated by noncompartmental analysis from plasma concentrations of midazolam, 1-hydroxymidazolam, and esaxerenone: primary endpoints (midazolam) AUC from zero to time of the last quantifiable concentration $\left(\mathrm{AUC}_{\text {last }}\right)$, AUC from zero to infinity $\left(\mathrm{AUC}_{\mathrm{inf}}\right)$, and peak plasma concentration $\left(C_{\max }\right)$; secondary endpoints (midazolam) - time to $C_{\max }\left(t_{\max }\right)$, elimination half-life $\left(t_{1 / 2}\right)$, apparent plasma clearance of drug after extravascular administration $(\mathrm{CL} / F)$, and apparent volume of distribution after extravascular administration $(V / F)$; secondary endpoints (1-hydroxymidazolam) $-\mathrm{AUC}_{\text {last }}, C_{\max }, t_{\max }$, and $\mathrm{AUC}_{\text {last }}$ and $C_{\max }$ ratio of 1-hydroxymidazolam to midazolam; and secondary endpoints (esaxerenone) $-C_{\max }$, AUC over the dosing interval $\left(\mathrm{AUC}_{\mathrm{tau}}\right)$, and $t_{\max }$ on day 1 ; and $C_{\text {max }}, \mathrm{AUC}_{\mathrm{tau}}, t_{\mathrm{max}}, t_{1 / 2}$, and accumulation ratio $\left(R_{\mathrm{ac}}\right)$ on day 14. For pharmacokinetic parameter calculations, Phoenix ${ }^{\circledR}$ WinNonlin ${ }^{\circledR}$ version 6.4 software (Certara USA Inc., Princeton, NJ, USA) was used. The $t_{1 / 2}$ of each subject was calculated based on the elimination rate constant (kel) in the terminal phase, and $\mathrm{AUC}_{\text {inf }}$ was calculated using the kel.

\subsection{Safety Assessment}

Safety endpoints comprised adverse events (AEs), laboratory tests, vital signs (body weight, BP, pulse rate, and body temperature), and electrocardiogram (ECG). The Medical Dictionary for Regulatory Activities (MedDRA) version
18.1 was used for coding of AEs by system organ class and preferred term.

\subsection{Statistical Analysis}

Sample size estimations were based on the literature for midazolam [29, 30], and assuming the individual variations in $\mathrm{AUC}_{\text {inf }}$ and $C_{\text {max }}$ (intraindividual coefficient of variation) to be 19 and $21 \%$, respectively, and that esaxerenone would not affect the pharmacokinetics of midazolam (geometric mean $[\mathrm{GM}]$ ratio 1.05$)$. Twenty-three subjects were estimated to be necessary to retain at least an $80 \%$ probability for two-sided $90 \%$ confidence intervals (CI) of GM ratios of $\mathrm{AUC}_{\text {inf }}$ and $C_{\max }$ to simultaneously fall within the range of $0.80-1.25$, which is generally considered to indicate that there is no pharmacokinetic interaction between drugs [26], assuming there are no interactions. The sample size was established as 28 subjects, in view of potential irregularities such as dropouts during the pharmacokinetic evaluation.

Tables of pharmacokinetic parameters for midazolam were prepared, and summary statistics for days 0 and 14 were calculated for the midazolam pharmacokinetic analysis set. All midazolam parameters were analysed based on a linear mixed model using the natural log-transformed value as the response variable, administration period as the fixed effect, and study subject as the random effect. For the response variable, the day 14 to day 0 ratio (and two-sided $90 \%$ CIs) of the geometric least squares mean was calculated by inverse log conversion of the least squares mean of the change calculated based on a linear mixed model.

Subjects in the midazolam pharmacokinetic analysis set were administered midazolam in both periods 1 and 2, and this set comprised all subjects without major protocol violations and for whom either of the primary study endpoints could be evaluated in both periods 1 and 2. Esaxerenone pharmacokinetic parameters were calculated for each day of administration in the esaxerenone pharmacokinetic analysis set, which comprised all subjects who had received at least one dose of esaxerenone and for whom esaxerenone plasma concentration data were available, and for whom no major protocol violations were documented. The safety analysis sets comprised data from all enrolled subjects given the study drug.

Statistical analyses and sample size calculations were performed using SAS System Release 9.2 software (SAS Institute Inc., Cary, NC, USA).

\section{Results}

\subsection{Study Subjects}

The study was conducted in 28 Japanese healthy males of mean (standard deviation [SD]) age 26.4 (4.5) years and 
mean (SD) BMI $20.5(1.3) \mathrm{kg} / \mathrm{m}^{2}$; other baseline characteristics for the safety analysis set $(n=28)$ are shown in Table 1 .

One subject was withdrawn from the study because of an $\mathrm{AE}$ and was excluded from the midazolam pharmacokinetic analysis set, and one was withdrawn because of use of a prohibited agent and was excluded from the midazolam and esaxerenone pharmacokinetic analysis sets. No subjects were excluded from the safety analysis set. Thus, the numbers of subjects included in the midazolam pharmacokinetic, esaxerenone pharmacokinetic, and safety analysis sets were 26 , 27 , and 28 , respectively.

\subsection{Pharmacokinetics}

Arithmetic mean (SD) values of midazolam $\mathrm{AUC}_{\text {last }}$ on days 0 and 14 were 18.7 (5.62) and 22.2 (6.22) $\mathrm{ng} \cdot \mathrm{h} / \mathrm{ml}$ for midazolam and midazolam with esaxerenone, respectively, and those of midazolam $\mathrm{AUC}_{\text {inf }}$ were 19.3 (5.68) and 22.9 (6.28) $\mathrm{ng} \cdot \mathrm{h} / \mathrm{ml}$, respectively (Table 2). The arithmetic mean (SD) plasma midazolam concentration increased slightly on day 14 compared with day $0\left(C_{\max } 8.19\right.$ [2.41] ng/ml on day 0 and 9.87 [2.37] ng/ml on day 14), as a result of repeated doses of $5 \mathrm{mg}$ esaxerenone once daily for 14 days, from days 1 to 14 (Table 2; Fig. 2). Analysis of geometric least squares mean ratios (midazolam with esaxerenone versus midazolam alone) revealed the following plasma midazolam pharmacokinetic ratios (two-sided 90\% CIs): AUC last $1.201(1.110,1.300), \mathrm{AUC}_{\text {inf }} 1.201(1.112,1.297)$, and $C_{\max } 1.224(1.116,1.342)$ (Table 3$)$. The arithmetic mean $t_{1 / 2}$ values on day $14(2.86 \mathrm{~h})$ and on day $0(2.63 \mathrm{~h})$ were similar, thus showing no differences between midazolam administered alone and in combination with esaxerenone (Table 2).

Plasma 1-hydroxymidazolam concentrations rapidly increased after a single dose of midazolam with or without esaxerenone, with a median $t_{\max }$ of $0.50 \mathrm{~h}$ in both groups and a decrease to below the lower limit of quantitation by $24 \mathrm{~h}$ after administration of midazolam in all subjects (Table 4). Plasma concentrations of 1-hydroxymidazolam increased with concomitant use of esaxerenone, and the respective arithmetic mean (SD) values of $\mathrm{AUC}_{\text {last }}$ on days 0 and 14 were $8.16(2.75)$ and $9.45(2.98) \mathrm{ng} \cdot \mathrm{h} / \mathrm{ml}$, and those of $C_{\max }$ were $4.07(1.60)$ and $4.78(1.46) \mathrm{ng} / \mathrm{ml}$. The arithmetic mean (SD) $\mathrm{AUC}_{\text {last }}$ ratio of 1-hydroxymidazolam to midazolam was $0.44(0.14)$ on day 0 and $0.41(0.11)$ on day 14 .

Table 1 Baseline characteristics (safety analysis set; $n=28$ )

\begin{tabular}{llc}
\hline Parameter & Mean (SD) & Range \\
\hline Age, years & $26.4(4.5)$ & $20-35$ \\
Bodyweight, $\mathrm{kg}$ & $60.5(5.7)$ & $50.7-71.7$ \\
Body mass index, $\mathrm{kg} / \mathrm{m}^{2}$ & $20.5(1.3)$ & $18.6-23.8$ \\
\hline
\end{tabular}

$S D$ standard deviation
Table 2 Pharmacokinetic parameters for midazolam alone and with esaxerenone in healthy Japanese males

\begin{tabular}{lll}
\hline Parameters & $\begin{array}{l}\text { Midazolam (day 0) } \\
n=26\end{array}$ & $\begin{array}{l}\text { Midazolam }+ \\
\text { esaxerenone } \\
\text { (day 14) } \\
n=26\end{array}$ \\
\hline $\mathrm{AUC}_{\text {last }}(\mathrm{ng} \cdot \mathrm{h} / \mathrm{ml})$ & $18.7(5.62)$ & $22.2(6.22)$ \\
$\mathrm{AUC}_{\mathrm{inf}}(\mathrm{ng} \cdot \mathrm{h} / \mathrm{ml})$ & $19.3(5.68)$ & $22.9(6.28)$ \\
$C_{\max }(\mathrm{ng} / \mathrm{ml})$ & $8.19(2.41)$ & $9.87(2.37)$ \\
$t_{1 / 2}(\mathrm{~h})$ & $2.63(0.596)$ & $2.86(0.857)$ \\
$t_{\max }(\mathrm{h})$ & $0.50(0.50,1.00)$ & $0.50(0.25,1.00)$ \\
$\mathrm{CL} / F(\mathrm{~L} / \mathrm{h})$ & $113.9(37.4)$ & $93.0(21.7)$ \\
$V / F(\mathrm{~L})$ & $416.2(107.8)$ & $377.2(127.3)$ \\
\hline
\end{tabular}

All values shown are arithmetic mean (standard deviation), except for $t_{\text {max }}$, which is listed as median (range)

$A U C_{\text {last }}$ area under curve from zero to time of the last quantifiable concentration, $A U C_{\text {inf }}$ mean area under the plasma concentration-time curve from zero to infinity, $C_{\max }$ mean peak plasma concentration, $t_{1 / 2}$ mean elimination half-life, $t_{\max }$ median time to $C_{\max }, C L / F$ apparent plasma clearance of drug after extravascular administration, $V / F$ apparent volume of distribution after extravascular administration

Plasma esaxerenone concentration increased rapidly after esaxerenone administration (Fig. 3): median $t_{\max }$ values on days 1 and 14 of administration were 3.0 and $2.5 \mathrm{~h}$, respectively, and the arithmetic mean (SD) of $t_{1 / 2}$ on day 14 was 18.10 (2.25) h (Table 5). Arithmetic mean (SD) values for esaxerenone $C_{\max }$ on days 1 and 14 were 66.75 (9.94) and $98.57(13.08) \mathrm{ng} / \mathrm{ml}$, respectively, and $\mathrm{AUC}_{\mathrm{tau}}$ values were $753.9(102.9)$ and $1322.0(197.0) \mathrm{ng} \cdot \mathrm{h} / \mathrm{ml}$, respectively. The $R_{\mathrm{ac}}$ during 14 days of repeated esaxerenone administration was 1.77 (Table 5).

\subsection{Safety}

One subject withdrew from the study (day 4, period 2) because of AEs: increased alanine aminotransferase (ALT), aspartate aminotransferase (AST), and lactate dehydrogenase (LDH). Six AEs occurred in three subjects (10.7\%; Table 6). AEs considered causally related to esaxerenone or midazolam (adverse reactions) comprised increased ALT, AST, and LDH, which occurred in a single subject during administration of esaxerenone alone. However, these reactions were mild, not associated with subjective symptoms, and resolved without intervention. There were no obvious differences in the incidence or type of AEs when midazolam was administered alone rather than coadministered with esaxerenone, and there were no clinically significant changes in laboratory test results, ECG findings, or vital signs. Overall, repeated administration of $5 \mathrm{mg} /$ day esaxerenone for 14 days in healthy Japanese adult males was not associated with any safety problems, and there were no 
Fig. 2 Mean (SD) plasma concentration-time profile of midazolam alone and with esaxerenone in healthy Japanese males (pharmacokinetic analysis set); inset shows the semi-log plot. $S D$ standard deviation

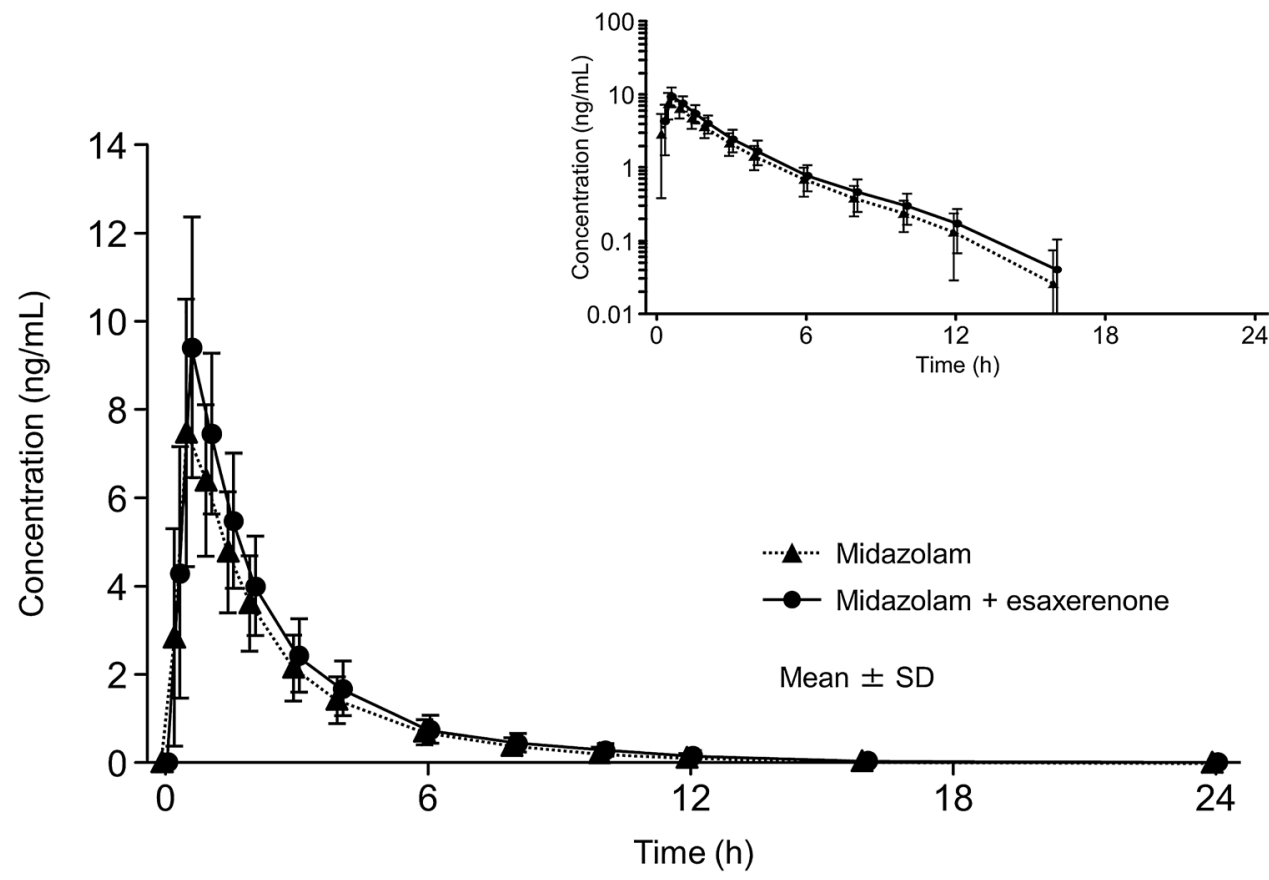

Table 3 Effects of esaxerenone on the pharmacokinetics of midazolam

\begin{tabular}{lllll}
\hline Parameters & $\begin{array}{l}\text { Mida- } \\
\text { zolam } \\
\text { day 0) } \\
n=26\end{array}$ & $\begin{array}{l}\text { Mida- } \\
\text { zolam + esaxer- } \\
\text { enone (day 14) } \\
n=26\end{array}$ & Ratio $^{\mathrm{a}}$ & 90\% CI \\
\hline $\mathrm{AUC}_{\text {last }}(\mathrm{ng} \cdot \mathrm{h} /$ & 17.9 & 21.5 & 1.201 & $1.110,1.300$ \\
$\mathrm{ml})$ & & & & \\
$\mathrm{AUC}_{\text {inf }}(\mathrm{ng} \cdot \mathrm{h} / \mathrm{ml})$ & 18.4 & 22.1 & 1.201 & $1.112,1.297$ \\
$C_{\max }(\mathrm{ng} / \mathrm{ml})$ & 7.85 & 9.61 & 1.224 & $1.116,1.342$ \\
\hline
\end{tabular}

All values shown are geometric least squares means

$C I$ confidence interval, $A U C_{\text {last }}$ area under curve from zero to time of the last quantifiable concentration, $A U C_{\text {inf }}$ mean area under the plasma concentration-time curve from zero to infinity, $C_{\max }$ mean peak plasma concentration

${ }^{\text {a }}$ Ratio: midazolam + esaxerenone to midazolam

safety concerns associated with concomitant administration of esaxerenone and midazolam.

\section{Discussion}

This study reveals that repeated administration of $5 \mathrm{mg} /$ day esaxerenone for 14 days produces an approximately 1.2 -fold increase in $C_{\max }$ and AUC of midazolam at $2 \mathrm{mg} / \mathrm{kg}$. The $t_{1 / 2}$ of midazolam and the metabolic ratio $\left(\mathrm{AUC}_{\text {last }}\right.$ ratio of
Table 4 Pharmacokinetic parameters for 1-hydroxymidazolam in healthy Japanese males

\begin{tabular}{|c|c|c|}
\hline Parameters & $\begin{array}{l}\text { Midazolam (day 0) } \\
n=26\end{array}$ & $\begin{array}{l}\text { Mida- } \\
\text { zolam }+ \text { esaxer- } \\
\text { enone (day 14) } \\
n=26\end{array}$ \\
\hline $\mathrm{AUC}_{\text {last }}(\mathrm{ng} \cdot \mathrm{h} / \mathrm{ml})$ & $8.16(2.75)$ & $9.45(2.98)$ \\
\hline$C_{\max }(\mathrm{ng} / \mathrm{ml})$ & $4.07(1.60)$ & $4.78(1.46)$ \\
\hline$t_{\max }(\mathrm{h})$ & $0.50(0.50,1.50)$ & $0.50(0.50,1.00)$ \\
\hline $\begin{array}{l}\mathrm{AUC}_{\text {last }} \text { ratio } \\
\text { (1-hydroxymida- } \\
\text { zolam to midazolam) }\end{array}$ & $0.44(0.14)$ & $0.41(0.11)$ \\
\hline $\begin{array}{l}C_{\max } \text { ratio (1-hydroxy- } \\
\text { midazolam to } \\
\text { midazolam) }\end{array}$ & $0.48(0.14)$ & $0.47(0.13)$ \\
\hline
\end{tabular}

All values shown are arithmetic mean (standard deviation), except for $t_{\max }$, which is listed as median (range)

$A U C_{\text {last }}$ area under curve from zero to time of the last quantifiable concentration, $C_{\max }$ mean peak plasma concentration, $t_{\max }$ median time to $C_{\max }$

1-hydroxymidazolam to midazolam) were unaffected by esaxerenone.

The lack of change in midazolam $t_{1 / 2}$ and the metabolic ratio, despite $C_{\max }$ and AUC increasing by approximately $20 \%$, when coadministered with esaxerenone suggests that increased exposure to midazolam results from increased bioavailability. In a previous study, neither the $t_{1 / 2}$ nor the metabolic ratio of midazolam increased when midazolam was coadministered with everolimus [31], suggesting that the bioavailability rather than the clearance is affected. 
Fig. 3 Mean (SD) plasma concentration-time profile of esaxerenone in healthy Japanese males on day 1 and day 14 (linear plots); inset shows the semi-log plot. $S D$ standard deviation

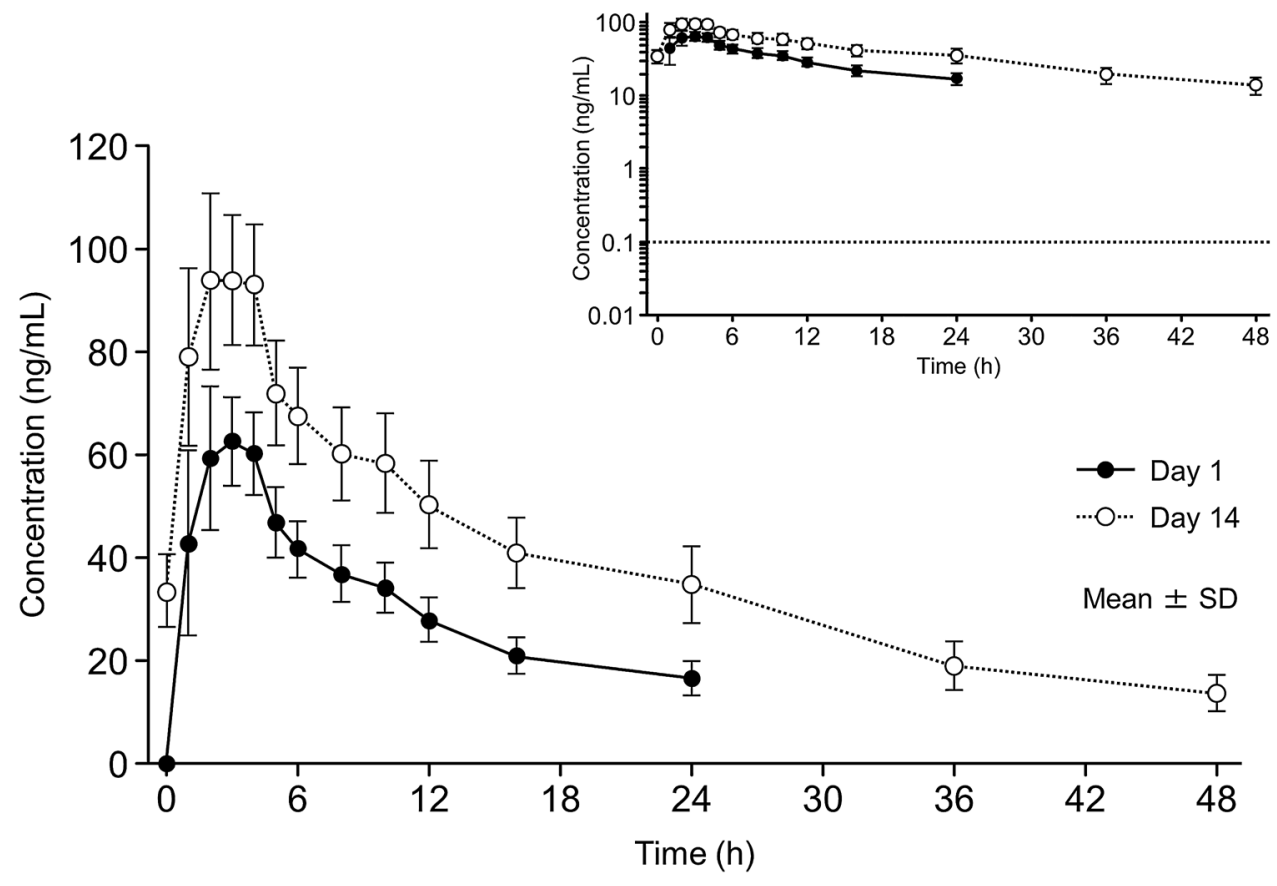

Table 5 Pharmacokinetic parameters for esaxerenone

\begin{tabular}{lll}
\hline Parameter & Day $1(n=27)$ & Day 14 $(n=26)$ \\
\hline$C_{\text {max }}(\mathrm{ng} / \mathrm{ml})$ & $66.75(9.94)$ & $98.57(13.08)$ \\
$\mathrm{AUC}_{\mathrm{tau}}(\mathrm{ng} \cdot \mathrm{h} / \mathrm{ml})$ & $753.9(102.9)$ & $1322.0(197.0)$ \\
$t_{\text {max }}(\mathrm{h})$ & $3.00(1.00,4.00)$ & $2.50(1.00,4.00)$ \\
$t_{1 / 2}(\mathrm{~h})$ & - & $18.10(2.25)$ \\
$R_{\mathrm{ac}}$ & - & $1.77(0.15)$ \\
\hline
\end{tabular}

All values shown are arithmetic means (standard deviations), except for $t_{\text {max }}$, which is listed as median (range)

$C_{\text {max }}$ mean peak plasma concentration, $A U C_{\text {tau }}$ area under curve over the dosing interval, $t_{\max }$ median time to $C_{\max }, t_{1 / 2}$ mean elimination half-life, $R_{a c}$ accumulation ratio

The main factors affecting bioavailability are drug fraction absorbed into enterocytes $\left(F_{\mathrm{a}}\right)$, intestinal availability $\left(F_{\mathrm{g}}\right.$; fraction escaping gut clearance), and hepatic availability $\left(F_{\mathrm{h}}\right.$; fraction escaping hepatic clearance). That is, bioavailability $(F)$ is the product of $F_{\mathrm{a}} \times F_{\mathrm{g}} \times F_{\mathrm{h}}$ [32]. In our study, for midazolam bioavailability to increase, one of the factors $\left(F_{\mathrm{a}}, F_{\mathrm{g}}\right.$, or $\left.F_{\mathrm{h}}\right)$ would have to increase. However, as esaxerenone has little influence on the absorption process $\left(F_{\mathrm{a}}\right)$ of midazolam, our data strongly suggest that midazolam $F_{\mathrm{g}}$ or $F_{\mathrm{h}}$ was increased by esaxerenone.

Several studies have outlined the importance of intestinal CYP3A in mediating clinically significant DDIs [33-35]. Pharmacokinetic estimation of the intestinal extraction of midazolam in healthy adults revealed an extraction ratio of 0.43 , which was similar to the hepatic extraction ratio (0.44), suggesting that intestinal CYP3A4 has a major influence on metabolism-based DDIs as well [33]. Indeed, the $F_{\text {a }}$ value for midazolam is close to 1 , and the extent of first-pass metabolism is relatively large. The intestinal and hepatic extraction are also relatively large. All of these factors indicate significant first-pass metabolism by intestinal and hepatic CYP3A after oral administration of midazolam.

Midazolam has a comparable first-pass effect in the liver and gastrointestinal tract [36]. However, in the current study, orally administered esaxerenone may have had a greater inhibitory effect against CYP3A in the gut rather than liver, as esaxerenone concentration in the gut will clearly be higher than in the liver immediately after oral administration. Moreover, esaxerenone is highly protein bound and its inhibitory activity against hepatic CYP3A is low. The intestinal inhibitory activity of esaxerenone against CYP3A may therefore have played a role in increasing midazolam bioavailability. However, this increase in AUC for a CYP3A-sensitive substrate (i.e. midazolam) was small (only 1.2-fold), thus suggesting limited potential for DDIs between esaxerenone and other CYP3A substrates. Indeed, DDI guidelines $[25,26]$ stipulate that a weak inhibitor of CYP3A is one that increases the AUC of a CYP3A substrate 1.25-2.0-fold. In the current study, esaxerenone did not meet this threshold criterion for even weak inhibition of CYP3A. In a previous study [37], esaxerenone increased the AUC of amlodipine, a substrate of CYP3A, by only about $20 \%$, which supports the results of this study.

Five $\mathrm{mg}$ /day esaxerenone, as used in this study, is approved as the maximum dosage for clinical use in Japan. A recently completed phase 3 study of esaxerenone (ESAXHTN; NCT02890173) compared dosages of 2.5 or $5 \mathrm{mg} /$ day with eplerenone $50 \mathrm{mg} /$ day in patients with essential 
Table 6 Adverse events

\begin{tabular}{|c|c|c|c|c|}
\hline & $\begin{array}{l}\text { Midazolam }^{\mathrm{a}} \\
n=28\end{array}$ & $\begin{array}{l}\text { Esaxerenone }^{\mathrm{b}} \\
n=28\end{array}$ & $\begin{array}{l}\text { Mida- } \\
\text { zolam }^{c}+\text { esaxer- } \\
\text { enone } \\
n=26\end{array}$ & $\begin{array}{l}\text { Total }^{\mathrm{d}} \\
n=28\end{array}$ \\
\hline Subjects with TEAEs & $0(0.0)$ & $3(10.7)$ & $0(0.0)$ & $3(10.7)$ \\
\hline Laboratory test abnormalities ${ }^{\mathrm{e}}$ & $0(0.0)$ & $3(10.7)$ & $0(0.0)$ & $3(10.7)$ \\
\hline Increased alanine aminotransferase ${ }^{f}$ & $0(0.0)$ & $1(3.6)$ & $0(0.0)$ & $1(3.6)$ \\
\hline Increased aspartate aminotransferase ${ }^{f}$ & $0(0.0)$ & $1(3.6)$ & $0(0.0)$ & $1(3.6)$ \\
\hline Increased blood lactate dehydrogenase ${ }^{f}$ & $0(0.0)$ & $1(3.6)$ & $0(0.0)$ & $1(3.6)$ \\
\hline Increased C-reactive protein & $0(0.0)$ & $2(7.1)$ & $0(0.0)$ & $2(7.1)$ \\
\hline
\end{tabular}

Data shown are number (\%) of patients. Some subjects experienced more than one laboratory test abnormality

TEAE treatment-emergent adverse event

${ }^{a}$ Tabulation category for treatment-emergent adverse events (TEAEs) collected after drug administration on day 0 and before drug administration on day 1

${ }^{\mathrm{b}}$ Tabulation category for TEAEs collected after drug administration on day 1 and before drug administration on day 14

${ }^{c}$ Tabulation category for TEAEs collected after drug administration on day 14

${ }^{\mathrm{d}}$ Tabulation category for TEAEs collected throughout the study

${ }^{\mathrm{e}}$ Medical Dictionary for Regulatory Activities/Japan; version 18.0

${ }^{\mathrm{f}}$ Occurred in the same subject

hypertension [17]. Results showed non-inferiority of $2.5 \mathrm{mg} /$ day esaxerenone when compared with $50 \mathrm{mg} /$ day eplerenone in terms of reduction of BP, and a superior antihypertensive effect for $5 \mathrm{mg} /$ day esaxerenone compared with $2.5 \mathrm{mg} /$ day esaxerenone and $50 \mathrm{mg} /$ day eplerenone. For ongoing research and development, this warrants investigation into lower esaxerenone dosages (e.g. 1.25 or $2.5 \mathrm{mg} /$ day) for potential clinical utility, and with attendant lower risks of CYP3A inhibition, such that any potential DDIs would unlikely be of clinical significance.

In the previous study, 39 healthy subjects were given $10-100 \mathrm{mg} /$ day for 10 days, and the mean pharmacokinetic values at day 10 with the $10 \mathrm{mg}$ /day dose were: $t_{\max } 2.5 \mathrm{~h}$, $t_{1 / 2} 25.1 \mathrm{~h}$, and $R_{\mathrm{ac}} 1.36$ [16]. The pharmacokinetic profile of esaxerenone during multiple administration in our study was similar to that in an earlier study [16]. Moreover, at day 10, the $C_{\max }$ and $\mathrm{AUC}_{\text {tau }}$ increased almost dose proportionally, and $t_{1 / 2}$ remained relatively constant within the range of 22-25 h [16]. In comparison, esaxerenone $t_{\max }$ remained relatively constant at $2.5-3.5 \mathrm{~h}$ and the $R_{\mathrm{ac}}$ was 1.77 over 14 days in the current study, during which plasma esaxerenone concentrations were considered to have reached steady-state.

Regarding esaxerenone safety in the current study, adverse reactions were mild and self-limiting. One subject withdrew because of an AE-a mild, transient increase in hepatic enzyme levels on day 4 of period 2, which was considered related to the study drug. As this AE did not cause any symptoms and resolved without treatment or sequelae 10 days after study drug discontinuation, this was not considered to be a major safety issue. As there were no clinically significant changes in vital signs, laboratory tests, or ECG findings, esaxerenone was not linked with any safety issues, and there were no safety concerns associated with concomitant administration of esaxerenone and midazolam.

The limitations of this study include the small sample size comprising only healthy Japanese male adults. Therefore, the safety data may not have enough statistical power. Another limitation is that in the evaluation of the pharmacokinetics of midazolam in this study. The magnitude of intra-individual variation in midazolam pharmacokinetics was not assessed; however, we believe the overall conclusions would remain unchanged. Finally, other DDI mechanisms that could cause issues through metabolic enzymes and transporters cannot be ruled out.

\section{Conclusion}

In summary, this study shows that, although there may be a low potential for DDIs with esaxerenone due to its CYP3Ainhibiting activity, at the clinically recommended dosage of $5 \mathrm{mg} /$ day, which is the approved maximum dosage for clinical use, midazolam AUC increased by only 1.2-fold and with no marked difference in $t_{1 / 2}$. This indicates no apparent potential for clinically significant DDIs between esaxerenone and CYP3A substrates, and no dose adjustment is necessary when drugs metabolised by CYP3A are used with esaxerenone. 
Acknowledgements The authors thank the following medical institution staff: Tatsuyuki Aihara, Naoko Suzuki, Saiko Yamada, and Kayo Matsumoto (clinical research coordinators); Yumie Aoki, Yuko Kotani, and Saeko Fukushima (nurses); and Dr Yasuko Owada (subinvestigator, Osaka Pharmacology Clinical Research Hospital). The authors thank Tsunenori Nakazawa (concentration measurer, Daiichi Sankyo Co., Ltd.) for his help with bioanalysis of the samples. The authors would like to acknowledge David Murdoch, BSc (Hons), of Edanz Pharma for medical writing support, which was funded by Daiichi Sankyo Co., Ltd.

\section{Declarations}

Funding This study was funded by Daiichi Sankyo Co., Ltd.

Conflicts of Interest KT, KK, TI, YO, TS, MK, YI, YN, and HI are employees of Daiichi Sankyo Co., Ltd. KK, TI, TS, YI, and HI own stocks in the company. HF has received research funding for this study from Daiichi Sankyo Co., Ltd.

Ethics approval The study was conducted after review and approval by the institutional review board of Osaka Pharmacology Clinical Research Hospital, Osaka, Japan (control number of the study site 930PC). The study was conducted in accordance with Good Clinical Practice and ethical principles of the Declaration of Helsinki and the Pharmaceuticals, Medical Devices, and OtherTherapeutic Products Act.

Informed Consent All subjects provided written informed consent to be included in the study (JapicCTI No. 152832).

Contributors KK, TI, and YO performed all statistical analyses. KT drafted the manuscript. HF conducted the study and collected the data. All authors contributed to the design and implementation of the research and provided critical feedback on the manuscript. All authors approved the final manuscript before submission.

Data Sharing Statement All de-identified patient data relevant to this study are included in this article. Additional data and supporting documents pertaining to this study are provided upon reasonable request made via this web address (https://vivli.org/ourmember/daiichi-san$\mathrm{kyo} /$ ) in accordance with the data sharing policy of Daiichi Sankyo Co., Ltd.

\section{Code Availability Not applicable.}

Consent for Publication Not applicable.

Open Access This article is licensed under a Creative Commons Attribution-NonCommercial 4.0 International License, which permits any non-commercial use, sharing, adaptation, distribution and reproduction in any medium or format, as long as you give appropriate credit to the original author(s) and the source, provide a link to the Creative Commons licence, and indicate if changes were made. The images or other third party material in this article are included in the article's Creative Commons licence, unless indicated otherwise in a credit line to the material. If material is not included in the article's Creative Commons licence and your intended use is not permitted by statutory regulation or exceeds the permitted use, you will need to obtain permission directly from the copyright holder. To view a copy of this licence, visit http://creativecommons.org/licenses/by-nc/4.0/.

\section{References}

1. Schmieder RE. End organ damage in hypertension. Dtsch Arztebl Int. 2010;107:866-73.

2. Perez MI, Musini VM. Pharmacological interventions for hypertensive emergencies: A Cochrane systematic review. J Hum Hypertens. 2008;22:596-607.

3. Umemura S, Arima H, Arima S, et al. The Japanese Society of Hypertension guidelines for the management of hypertension (JSH 2019). Hypertens Res. 2019;42:1235-481.

4. Chobanian AV, Bakris GL, Black HR, et al. Seventh report of the Joint National Committee on prevention, detection, evaluation, and treatment of high blood pressure. Hypertension. 2003;42:1206-52.

5. Tedesco MA, Natale F, Calabro R. Effects of monotherapy and combination therapy on blood pressure control and target organ damage: a randomized prospective intervention study in a large population of hypertensive patients. J Clin Hypertens (Greenwich). 2006;8:634-41.

6. Hozawa A, Ohkubo T, Kikuya M, et al. Blood pressure control assessed by home, ambulatory and conventional blood pressure measurements in the Japanese general population: the Ohasama study. Hypertens Res. 2002;25:57-63.

7. Epstein M, Duprez DA. Resistant hypertension and the pivotal role for mineralocorticoid receptor antagonists: a clinical update 2016. Am J Med. 2016;129:661-6.

8. Takahashi S, Katada J, Daida H, Kitamura F, Yokoyama K. Effects of mineralocorticoid receptor antagonists in patients with hypertension and diabetes mellitus: a systematic review and metaanalysis. J Hum Hypertens. 2016;30:534-42.

9. Narayan H, Webb DJ. New evidence supporting the use of mineralocorticoid receptor blockers in drug-resistant hypertension. Curr Hypertens Rep. 2016;18:34.

10. Lainscak M, Pelliccia F, Rosano G, et al. Safety profile of mineralocorticoid receptor antagonists: spironolactone and eplerenone. Int J Cardiol. 2015;200:25-9.

11. Duggan S. Esaxerenone: First global approval. Drugs. 2019;79:477-81.

12. Arai K, Tsuruoka H, Homma T. CS-3150, a novel non-steroidal mineralocorticoid receptor antagonist, prevents hypertension and cardiorenal injury in Dahl salt-sensitive hypertensive rats. Eur J Pharmacol. 2015;769:266-73.

13. Arai K, Morikawa Y, Ubukata N, Tsuruoka H, Homma T. CS-3150, a novel nonsteroidal mineralocorticoid receptor antagonist, shows preventive and therapeutic effects on renal injury in deoxycorticosterone acetate/salt-induced hypertensive rats. J Pharmacol Exp Ther. 2016;358:548-57.

14. Li L, Guan Y, Kobori H, et al. Effects of the novel nonsteroidal mineralocorticoid receptor blocker, esaxerenone (CS-3150), on blood pressure and urinary angiotensinogen in low-renin Dahl salt-sensitive hypertensive rats. Hypertens Res. 2019;42:769-78.

15. Bhuiyan AS, Rafiq K, Kobara H, Masaki T, Nakano D, Nishiyama A. Effect of a novel nonsteroidal selective mineralocorticoid receptor antagonist, esaxerenone (CS-3150), on blood pressure and renal injury in high salt-treated type 2 diabetic mice. Hypertens Res. 2019;42:892-902.

16. Kato M, Furuie H, Shimizu T, Miyazaki A, Kobayashi F, Ishizuka H. Single- and multiple-dose escalation study to assess pharmacokinetics, pharmacodynamics and safety of oral esaxerenone in healthy Japanese subjects. Br J Clin Pharmacol. 2018;84:1821-9.

17. Ito S, Ito H, Rakugi H, Okuda Y, Yoshimura M, Yamakawa S. A double-blind phase III study of esaxerenone (CS-3150) compared to eplerenone in patients with essential hypertension (ESAX-HTN study). Hypertension. 2020;75:51-8. 
18. Katoh M, Nakajima M, Shimada N, Yamazaki H, Yokoi T. Inhibition of human cytochrome $\mathrm{P} 450$ enzymes by 1,4-dihydropyridine calcium antagonists: prediction of in vivo drug-drug interactions. Eur J Clin Pharmacol. 2000;55:843-52.

19. Ma B, Prueksaritanont T, Lin JH. Drug interactions with calcium channel blockers: possible involvement of metabolite-intermediate complexation with CYP3A. Drug Metab Dispos. 2000;28:125-30.

20. Siller-Matula JM, Lang I, Christ G, Jilma B. Calcium-channel blockers reduce the antiplatelet effect of clopidogrel. J Am Coll Cardiol. 2008;52:1557-63.

21. Yoshida M, Matsumoto T, Suzuki T, Kitamura S, Mayama T. Effect of concomitant treatment with a CYP3A4 inhibitor and a calcium channel blocker. Pharmacoepidemiol Drug Saf. 2008;17:70-5.

22. Zisaki A, Miskovic L, Hatzimanikatis V. Antihypertensive drugs metabolism: an update to pharmacokinetic profiles and computational approaches. Curr Pharm Des. 2015;21:806-22.

23. Yamada M, Ishizuka T, Inoue SI, Rozehnal V, Fischer T, Sugiyama D. Drug-drug interaction risk assessment of esaxerenone as a perpetrator by in vitro studies and static and physiologically based pharmacokinetic models. Drug Metab Dispos. 2020;48:769-77.

24. Galetin A, Ito K, Hallifax D, Houston JB. CYP3A4 substrate selection and substitution in the prediction of potential drug-drug interactions. J Pharmacol Exp Ther. 2005;314:180-90.

25. US Department of Health and Human Services, Food and Drug Administration, Center for Drug Evaluation and Research (CDER). Clinical drug interaction studies-Cytochrome P450 Enzyme- and Transporter-Mediated Drug Interactions Guidance for Industry. Guidance document, 2017. https://www.fda.gov/ media/134581/download. Accessed 23 Jun 2021.

26. Ministry of Health, Labour and Welfare, Tokyo, Japan; 2017. Guideline on drug interactions for drug development and information provision (final draft), PFSB/ELD Notice. https://www. pmda.go.jp/files/000225191.pdf. Accessed 23 Jun 2021.

27. Thummel KE, Shen DD, Podoll TD, et al. Use of midazolam as a human cytochrome $\mathrm{P} 450$ 3A probe: $\mathrm{I}$. In vitro-in vivo correlations in liver transplant patients. J Pharmacol Exp Ther. 1994;271:549-56.
28. Paulson SK, Wood-Horrall RN, Hoover R, Quintas M, Lawrence LE, Cammarata SK. The pharmacokinetics of the CYP3A substrate midazolam after steady-state dosing of delafloxacin. Clin Ther. 2017;39:1182-90.

29. Zhou H, Tong Z, McLeod JF. "Cocktail" approaches and strategies in drug development: valuable tool or flawed science? J Clin Pharmacol. 2004;44:120-34.

30. Jang G, Kaufman A, Lee E, et al. A clinical therapeutic protein drug-drug interaction study: coadministration of denosumab and midazolam in postmenopausal women with osteoporosis. Pharmacol Res Perspect. 2014;2:e00033.

31. Urva S, Bouillaud E, Delaney R, Jappe A, Cheung W. A phase I study evaluating the effect of everolimus on the pharmacokinetics of midazolam in healthy subjects. J Clin Pharmacol. 2013;53:444-50.

32. Chaturvedi PR, Decker CJ, Odinecs A. Prediction of pharmacokinetic properties using experimental approaches during early drug discovery. Curr Opin Chem Biol. 2001;5:452-63.

33. Thummel KE, O'Shea D, Paine MF, et al. Oral first-pass elimination of midazolam involves both gastrointestinal and hepatic CYP3A-mediated metabolism. Clin Pharmacol Ther. 1996;59:491-502.

34. Gorski JC, Jones DR, Haehner-Daniels BD, Hamman MA, O'Mara EM Jr, Hall SD. The contribution of intestinal and hepatic CYP3A to the interaction between midazolam and clarithromycin. Clin Pharmacol Ther. 1998;64:133-43.

35. Qiu F, Wang G, Zhang R, Sun J, Jiang J, Ma Y. Effect of danshen extract on the activity of CYP3A4 in healthy volunteers. Br J Clin Pharmacol. 2010;69:656-62.

36. Shen DD, Kunze KL, Thummel KE. Enzyme-catalyzed processes of first-pass hepatic and intestinal drug extraction. Adv Drug Deliv Rev. 1997;27:99-127.

37. Kirigaya Y, Shiramoto M, Ishizuka T, et al. Pharmacokinetic interactions of esaxerenone with amlodipine and digoxin in healthy Japanese subjects. BMC Pharmacol Toxicol. 2020;21:55. 